\title{
25. INTERLABORATORY COMPARISON OF LEG 92 STANDARD BASALT SAMPLE ANALYSES ${ }^{1}$
}

\author{
Jörg Erzinger, Mineralogisch-Petrologisches Institut, Justus-Liebig Universität ${ }^{2}$
}

\section{INTRODUCTION}

Two basalts recovered from Hole 597C were selected to be part of an interlaboratory comparison of geochemical analyses being performed by laboratories contributing analytical data to this volume.

\section{SAMPLE PREPARATION}

The two samples selected were from massive basalt flows drilled in Hole 597C: Sample 597C-4-5, 120-130 cm (our Sample ILB 1) and Sample 597C-8-2, 85-96 cm (our Sample ILB 2). The samples are relatively fresh with respect to alteration-sensitive elements (Erzinger, this volume), and they have compositions that are generally representative of Hole 597C basement.

The samples were prepared as follows: after being washed with distilled water, dried, and crushed, the samples were ground in an agate mill and homogenized by repeated splitting. Portions weighing about $10 \mathrm{~g}$ each were sent to nine laboratories performing geochemical analyses of Leg 92 basalts.

\section{RESULTS OF ANALYSES}

Four laboratories returned results, which are summarized in Tables 1 and 2 . The code (used in tables), the location of the lab, the principal investigator (or analyst), and the submitted comments on the analytical methods are as follows. The code UMUS indicates the analysis from the University of Minnesota done by R. Knoche. The samples were dried at $110^{\circ} \mathrm{C}$ prior to analysis. The analyses were done by direct-current plasma optical emission spectroscopy from $\mathrm{LiBO}_{2}$ fussion/ $\mathrm{HCl}$ dissolution; $\mathrm{FeO}$ was determined by titration (the "Wilson" method). The code UNUK indicates the analysis from the University of Newcastle upon Tyne, U.K., done by J. Pearce. The elements Hf, Ta, Th, Sc, Co, La, Ce, Nd, $\mathrm{Sm}, \mathrm{Eu}, \mathrm{Tb}, \mathrm{Ho}, \mathrm{Tm}, \mathrm{Yb}$, and $\mathrm{Lu}$ were analyzed by INAA, all others by X-ray fluorescence. Data were calculated on a dry-weight basis. The code CNEXO indicates the analysis from the Centre National pour l'Exploitation des Océans, France, done by J. Etoubleau. The results were obtained on dried samples $\left(105^{\circ} \mathrm{C}\right)$ by $\mathrm{X}$-ray fluorescence. The code UGFRG indicates the analysis from the University of Giessen, F.R.G., done by J. Erzinger and $\mathrm{M}$. Grünhäuser. The samples were dried at $110^{\circ} \mathrm{C}$ prior to analysis. The major oxides and some trace elements (Co, Cr, Cu, Ga, Ni, Rb, Sr, Y, Zn, and $\mathrm{Zr}$ )

\footnotetext{
${ }^{1}$ Leinen, M., Rea, D. K., et al., Init. Repts. DSDP, 92: Washington (U.S. Govt. Printing Office).

2 Address: Mineralogisch-Petrologisches Institut, Justus-Liebig Universität, Senckenbergstrasse 3, D-6300 Giessen, Federal Republic of Germany.
}

Table 1. Chemical composition of Leg 92 "standard" basalt Sample 597C-4-5, 120-130 cm (ILB 1).

\begin{tabular}{|c|c|c|c|c|c|c|c|c|}
\hline & UMUS & sd & UNUK & sd & CNEXO & UGFRG & sd & $N$ \\
\hline $\mathrm{SiO}_{2}(\mathrm{wt}, \%)$ & 50.8 & 0.6 & 49.77 & 0.26 & 49.83 & 50.44 & 0.07 & 30 \\
\hline $\mathrm{TiO}_{2}$ & 1.35 & 0.02 & 1.32 & 0.01 & 1.33 & 1.328 & 0.004 & 30 \\
\hline $\mathrm{Al}_{2} \mathrm{O}_{3}$ & 14.8 & 0.2 & 14.60 & 0.19 & 14.56 & 14.83 & 0.04 & 30 \\
\hline $\mathrm{Fe}_{2} \mathrm{O}_{3}$ & 3.4 & 0.1 & - & - & - & 3.39 & - & - \\
\hline $\mathrm{FeO}^{\circ}$ & 7.6 & 0.1 & - & - & - & 7.67 & - & 2 \\
\hline $\mathrm{MnO}$ & 0.164 & 0.003 & 0.16 & 0.00 & 0.16 & 0.166 & 0.002 & 30 \\
\hline $\mathrm{MgO}$ & 7.34 & 0.007 & 7.40 & 0.14 & 7.33 & 7.39 & 0.02 & 30 \\
\hline $\mathrm{CaO}$ & 11.8 & 0.2 & 11.78 & 0.06 & 11.92 & 11.85 & 0.014 & 30 \\
\hline $\mathrm{Na}_{2} \mathrm{O}$ & 2.44 & 0.04 & 2.39 & 0.30 & 2.21 & 2.44 & 0.02 & 30 \\
\hline $\mathrm{K}_{2} \mathrm{O}$ & 0.061 & 0.005 & 0.09 & 0.01 & 0.09 & 0.097 & 0.001 & 30 \\
\hline $\mathrm{P}_{2} \mathrm{O}_{5}$ & 0.104 & 0.004 & 0.13 & 0.03 & 0.13 & 0.110 & 0.003 & 30 \\
\hline $\mathrm{H}_{2}^{-} \mathrm{O}^{+}$ & - & - & - & - & - & 0.42 & 0.01 & 5 \\
\hline $\mathrm{CO}_{2}$ & - & - & $\vec{a}$ & - & - & 0.04 & - & 4 \\
\hline Lố & - & - & 0.41 & - & 0.34 & - & - & - \\
\hline Total & 99.86 & - & 99.52 & - & 99.81 & 100.175 & - & - \\
\hline $\mathrm{Fe}_{2} \mathrm{O}_{3}$ & 11.8 & 0.2 & 11.62 & 0.05 & 11.91 & 11.91 & 0.03 & 30 \\
\hline $\mathrm{Ba}(\mathrm{ppm})$ & 12.2 & 0.5 & - & - & - & - & - & - \\
\hline $\mathrm{Be}$ & 0.4 & 0.1 & - & - & - & - & - & - \\
\hline Co & 42 & 2 & 43.1 & - & 44 & 43 & 2.5 & 24 \\
\hline $\mathrm{Cr}$ & 197 & 3 & 215 & 10 & 200 & 198 & 2.3 & 24 \\
\hline $\mathrm{Cu}$ & 136 & 3 & 121 & 4.2 & - & 122 & 1.1 & 24 \\
\hline Ga & 17 & 2 & - & - & - & 17 & 0.8 & 24 \\
\hline $\mathrm{Hf}$ & 5 & 2 & 2.03 & - & - & - & - & - \\
\hline Mo & $<2$ & 2 & - & - & - & - & - & - \\
\hline $\mathrm{Nb}$ & - & - & 4.5 & 0.3 & 3.7 & 1 & 0.6 & 42 \\
\hline $\mathrm{Ni}$ & 63 & 2 & 78 & 9.0 & 67 & 62 & 1.1 & 24 \\
\hline $\mathrm{Pb}$ & $<5$ & 5 & - & - & - & - & - & - \\
\hline $\mathrm{Rb}$ & $<1$ & 1 & 3 & 0.3 & 0 & $<5$ & - & 42 \\
\hline Sc & 44 & 1 & 46.2 & - & - & - & - & - \\
\hline Sr & 88 & 3 & 87 & 0.8 & 87 & 94 & 0.7 & 42 \\
\hline Ta & - & - & 0.23 & - & - & - & - & - \\
\hline Th & $<5$ & 5 & 0.30 & - & - & - & - & - \\
\hline v & 345 & 5 & 346 & 16 & 369 & - & - & - \\
\hline Y & 33 & 1 & 31 & 0.4 & 31 & 30 & 0.5 & 42 \\
\hline $\mathrm{Zn}$ & 97 & 3 & 89 & 0.6 & 70 & 90 & 1.0 & 24 \\
\hline $\mathrm{Zr}$ & 75 & 5 & 79 & 0.7 & 78 & 87 & 0.8 & 42 \\
\hline La & - & - & 3.2 & - & - & 3.21 & 0.16 & - \\
\hline $\mathrm{Ce}$ & - & - & 9.2 & - & - & 9.22 & 0.85 & - \\
\hline Pr & - & - & - & - & - & 2.03 & 0.18 & - \\
\hline $\mathrm{Nd}$ & - & - & 8.9 & - & - & 7.4 & 1.1 & - \\
\hline Sm & - & - & 2.97 & - & - & 2.75 & 0.19 & - \\
\hline Eu & - & - & 1.12 & - & - & 1.06 & 0.11 & - \\
\hline Gd & - & - & - & - & - & 5.03 & 0.95 & - \\
\hline Tb & - & - & 0.81 & - & - & 0.58 & 0.05 & - \\
\hline Dy & - & - & - & - & - & 4.97 & 0.42 & - \\
\hline Ho & - & - & 1.48 & - & - & 0.87 & 0.06 & - \\
\hline Er & - & - & - & - & - & 3.21 & 0.3 & - \\
\hline $\mathrm{Tm}$ & - & - & 0.52 & - & - & 0.43 & 0.11 & - \\
\hline Yb & - & - & 3.24 & - & - & 3.07 & 0.30 & - \\
\hline Lu & - & - & 0.56 & - & - & 0.45 & 0.05 & - \\
\hline
\end{tabular}

Note: $s d=$ standard deviation; $N=$ number of measurements; - indicates not determined or below detection limit. Total iron as $\mathrm{Fe}_{2} \mathrm{O}_{3} . \mathrm{LOI}=$ loss on ignition.

were analyzed by X-ray fluorescence with fused glass disks or pressed powder pellets, respectively. Ferrous iron analysis was performed by manganometric titration, $\mathrm{H}_{2} \mathrm{O}^{+}$ by coulometric Karl-Fischer titration, and $\mathrm{CO}_{2}$ coulometrically following thermal decomposition. Rare-earth elements were done by inductively coupled plasma optical emission spectroscopy.

Mean values were not calculated. It is evident from Tables 1 and 2 that the agreement between the laboratories is unusually good.

Date of Initial Receipt: 25 September 1984 Date of Acceptance: 29 December 1984 
Table 2. Chemical composition of Leg 92 "standard" basalt Sample 597C-8-2, 85-96 cm (ILB 2).

\begin{tabular}{|c|c|c|c|c|c|c|c|}
\hline & UMUS & sd & UNUK & CNEXO & UGFRG & sd & $\mathrm{N}$ \\
\hline $\mathrm{SiO}_{2}(w \mathrm{wt} . \%)$ & 50.8 & 0.6 & 49.95 & 50.50 & 50.45 & 0.07 & 29 \\
\hline $\mathrm{TiO}_{2}$ & 1.50 & 0.02 & 1.47 & 1.52 & 1.487 & 0.003 & 29 \\
\hline $\mathrm{Al}_{2} \mathrm{O}_{3}$ & 13.8 & 0.2 & 13.64 & 13.58 & 13.78 & 0.03 & 29 \\
\hline $\mathrm{Fe}_{2} \mathrm{O}_{3}$ & 3.6 & 0.1 & - & - & 4.15 & - & - \\
\hline $\mathrm{FeO}$ & 8.6 & 0.1 & - & - & 8.31 & - & 3 \\
\hline $\mathrm{MnO}$ & 0.196 & 0.003 & 0.19 & 0.19 & 0.199 & 0.002 & 29 \\
\hline $\mathrm{MgO}$ & 7.25 & 0.007 & 7.50 & 7.21 & 7.31 & 0.03 & 29 \\
\hline $\mathrm{CaO}$ & 11.1 & 0.2 & 11.20 & 11.37 & 11.22 & 0.01 & 29 \\
\hline $\mathrm{Na}_{2} \mathrm{O}$ & 2.50 & 0.04 & 2.16 & 2.27 & 2.49 & 0.024 & 29 \\
\hline $\mathrm{K}_{2} \mathrm{O}$ & 0.116 & 0.005 & 0.14 & 0.10 & 0.126 & 0.001 & 29 \\
\hline $\mathrm{P}_{2} \mathrm{O}_{5}$ & 0.121 & 0.004 & 0.12 & 0.14 & 0.125 & 0.002 & 29 \\
\hline $\mathrm{H}_{2} \mathrm{O}^{+}$ & - & - & - & - & 0.52 & 0.01 & 4 \\
\hline $\mathrm{CO}_{2}$ & - & - & - & - & 0.03 & - & 4 \\
\hline LOI & 一 & - & 0.42 & 0.31 & - & 一 & - \\
\hline Total & 99.58 & - & 99.76 & 100.49 & 100.193 & - & - \\
\hline $\mathrm{Fe}_{2} \mathrm{O}_{3}$ & 13.2 & 0.2 & 12.97 & 13.30 & 13.38 & 0.04 & 29 \\
\hline $\mathrm{Ba}$ (ppm) & 10.3 & 0.5 & - & - & - & - & 一 \\
\hline $\mathrm{Be}$ & 0.5 & 0.1 & - & - & - & - & - \\
\hline Co & 46 & 2 & - & 46 & 48 & 2.1 & 12 \\
\hline $\mathrm{Cr}$ & 97 & 3 & 211 & 110 & 122 & 1.5 & 12 \\
\hline $\mathrm{Cu}$ & 149 & 3 & 130 & - & 126 & 0.9 & 12 \\
\hline Ga & 15 & 2 & - & - & 17 & 0.7 & 12 \\
\hline Hf & $<2$ & 2 & - & - & - & - & 一 \\
\hline Mo & $<2$ & 2 & - & - & - & - & - \\
\hline $\mathrm{Nb}$ & - & - & 4.5 & 3.3 & 1.2 & 0.5 & 21 \\
\hline $\mathrm{Ni}$ & 55 & 2 & 82 & 42 & 51 & 0.7 & 12 \\
\hline $\mathrm{Pb}$ & $<5$ & 5 & - & - & - & - & - \\
\hline $\mathrm{Rb}$ & 2 & 1 & 5.5 & 0.9 & $<5$ & - & 21 \\
\hline Sc & 46 & 1 & - & - & - & - & - \\
\hline $\mathrm{Sr}$ & 82 & 3 & 83 & 82 & 89 & 0.7 & 21 \\
\hline Ta & - & - & - & - & - & - & - \\
\hline Th & 6 & 5 & - & - & - & - & - \\
\hline V & 390 & 5 & 353 & 395 & - & - & - \\
\hline Y & 35 & 1 & 34 & 35 & 32 & 0.5 & 21 \\
\hline $\mathrm{Zn}$ & 105 & 3 & 90 & 82 & 101 & 1.1 & 12 \\
\hline $\mathrm{Zr}$ & 84 & 5 & 88 & 85 & 95 & 1.0 & 21 \\
\hline $\mathrm{La}$ & - & - & - & - & 3.53 & 0.3 & - \\
\hline $\mathrm{Ce}$ & - & - & - & - & 10.2 & 1.0 & - \\
\hline $\mathrm{Pr}$ & - & - & - & - & 2.19 & 0.02 & - \\
\hline Nd & - & - & - & - & 8.56 & 0.99 & - \\
\hline $\mathrm{Sm}$ & - & - & - & - & 3.74 & 0.23 & - \\
\hline $\mathrm{Eu}$ & - & - & - & - & 1.23 & 0.03 & - \\
\hline Gd & - & - & - & - & - & - & - \\
\hline $\mathrm{Tb}$ & - & - & - & - & 0.73 & 0.06 & - \\
\hline Dy & - & - & - & - & 5.82 & 0.1 & - \\
\hline Ho & - & - & - & - & 0.94 & 0.07 & - \\
\hline Er & - & - & - & - & 3.57 & 0.12 & - \\
\hline Tm & - & - & - & - & 0.44 & 0.08 & - \\
\hline $\mathrm{Yb}$ & - & - & - & - & 3.25 & 0.1 & - \\
\hline Lu & - & - & - & - & 0.48 & 0.02 & - \\
\hline
\end{tabular}

Note: s.d. = standard deviation; $\mathrm{N}=$ number of measurements; - indicates not determined or below detection limit. Total iron as $\mathrm{Fe}_{2} \mathrm{O}_{3}$. LOI $=$ loss on ignition. 